\title{
A fresh look at Euler's limit formula for the gamma function
}

\section{G. J. O. JAMESON}

\section{Introduction}

Consider the problem of defining a continuous function $f(x)$ which agrees with factorials at integers. There are many possible ways to do this. In fact, such a function can be constructed by taking any continuous definition of $f(x)$ on $[0,1]$ with $f(0)=f(1)=1$ (such as $f(x)=1$ ), and then extending the definition to all $x>1$ by the formula $f(x+1)=(x+1) f(x)$. This construction was discussed by David Fowler in [1] and [2]. For example, the choice $f(x)=\frac{1}{2} x(x-1)+1$ results in a function that is differentiable everywhere, including at integers.

However, this approach had already been overtaken in 1729 , when Euler obtained the conclusive solution to the problem by defining what we now call the gamma function. Among all the possible functions that reproduce factorials, this is the 'right' one, in the sense that it is the only one satisfying a certain smoothness condition which we will specify below. Admittedly, Euler didn't know this. It is known as the Bohr-Mollerup theorem, and was only proved nearly two centuries later.

First, a remark on notation: the notation $\Gamma(x)$ for the gamma function, introduced by Legendre, is such that $\Gamma(n)$ is actually $(n-1)$ ! instead of $n$ !. Though this might seem a little perverse, it does result in some formulae becoming slightly neater. Some writers, including Fowler, write $x$ ! for $\Gamma(x+1)$, and refer to this as the 'factorial function'. However, the notation $\Gamma(x)$ is very firmly entrenched, and I will adhere to it here.

Euler actually introduced two completely different ways to define the gamma function, which turn out to be equivalent. One is by the (now very well-known) integral

$$
\int_{0}^{\infty} t^{x-1} e^{-t} d t
$$

which converges, so can be taken as the definition of $\Gamma(x)$, for all $x>0$.

Our subject here is Euler's other definition. It is perhaps a little less well known, but it still plays an essential part in the theory of the gamma function. It takes the form of a certain limit. How did Euler think of it? He started by rewriting factorials as follows. For integers $k$ and $n$,

$$
(k-1) !=\frac{(n+k) !}{k(k+1) \ldots(n+k)}
$$

and

$$
\frac{(n+k) !}{n^{k} n !}=\frac{(n+1)(n+2) \ldots(n+k)}{n^{k}}=\left(1+\frac{1}{n}\right)\left(1+\frac{2}{n}\right) \ldots\left(1+\frac{k}{n}\right) .
$$

Denote this by $C(n, k)$. Then $C(n, k) \rightarrow 1$ as $n \rightarrow \infty$ and 
$(k-1) !=C(n, k) \Gamma_{n}(k)$, where

$$
\Gamma_{n}(k)=\frac{n^{k} n !}{k(k+1) \ldots(k+n)} \text {. }
$$

So $\Gamma_{n}(k) \rightarrow(k-1)$ ! as $n \rightarrow \infty$. To extend to non-integers, we now define (for any $x$ except 0 and negative integers)

$$
\Gamma_{n}(x)=\frac{n^{x} n !}{x(x+1) \ldots(x+n)} .
$$

and then define $\Gamma(x)$ to be $\lim _{n \rightarrow \infty} \Gamma_{n}(x)$.

However, to do this, we need to show that the limit exists. In most accounts of the gamma function (e.g. [3], [4], [5]), this is done by establishing its equivalence to a certain infinite product, and then proving the convergence of this product. This procedure has the merit of applying equally to real or complex $x$. Here I will describe an alternative method for the real case (not, as far as I know, to be found anywhere in the literature), based on the well-known elementary fact that a sequence is convergent if it is increasing and bounded above. As well as being pleasantly simple, this method has the bonus that it delivers, for free, very effective upper and lower bounds for $\Gamma(x)$. It also paves the way for the proof of the BohrMollerup theorem by a neat reversal of the same reasoning.

\section{Convergence of the limit}

Instead of $\Gamma_{n}(x)$ itself, we work with two slight variants:

$$
\begin{aligned}
& G_{n}(x)=\frac{n^{x-1} n !}{x(x+1) \ldots(x+n-1)}, \\
& H_{n}(x)=\frac{(n+1)^{x-1} n !}{x(x+1) \ldots(x+n-1)} .
\end{aligned}
$$

The numerator $n^{x-1} n$ ! in $G_{n}(x)$ can equally be written as $n^{x}(n-1)$ !. We have

$$
\Gamma_{n}(x)=\frac{n}{x+n} G_{n}(x), \quad H_{n}(x)=\left(1+\frac{1}{n}\right)^{x-1} G_{n}(x),
$$

so if any of the three sequences converges, then so do the others, with the same limit. Also, $\Gamma_{n}(x)<G_{n}(x)$ for all $x>0$, and $G_{n}(x)<H_{n}(x)$ for $x>1$, while $G_{n}(x)>H_{n}(x)$ for $0<x<1$. Note that $G_{n}(1)=H_{n}(1)=1$ (also $\left.H_{n}(2)=1\right)$, while $\Gamma_{n}(1)=n /(n+1)$, suggesting that in some sense $G_{n}$ and $H_{n}$ are more 'natural' than $\Gamma_{n}$. Note also that $x \Gamma_{n}(x)=G_{n}(x+1)$.

We use the following well-known inequality:

Lemma 1: For all $t>0,(1+t)^{p}>1+p t$ if $p>1$, and $(1+t)^{p}<1+p t$ if $0<p<1$. 
Proof: By the mean-value theorem, applied to $f(t)=(1+t)^{p}$, we have

$$
(1+t)^{p}-1=f(t)-f(0)=p t(1+u)^{p-1}
$$

for some $u$ in $(0, t)$. Clearly, $(1+u)^{p-1}>1$ if $p>1$, and the reverse holds if $0<p<1$.

The key is the following monotonic behaviour of $G_{n}(x)$ and $H_{n}(x)$, switching at certain values of $x$.

\section{Theorem 1:}

(i) $G_{n}(x)$ increases (strictly) with $n$ for $x>1$, and decreases for $0<x<1$.

(ii) $H_{n}(x)$ increases with $n$ for $x>2$ and for $0<x<1$, and decreases for $1<x<2$.

Proof:

(i) Let $\delta_{n}(x)=G_{n+1}(x) / G_{n}(x)$. Then

$$
\delta_{n}(x)=\left(\frac{n+1}{n}\right)^{x} \frac{n}{n+x}=\left(1+\frac{1}{n}\right)^{x}\left(1+\frac{x}{n}\right)^{-1}
$$

By Lemma 1 , for $x>1$ we have $\left(1+\frac{1}{n}\right)^{x}>1+\frac{x}{n}$, so $\delta_{n}(x)>1$, and similarly $\delta_{n}(x)<1$ for $0<x<1$.

(ii) Now let $\mu_{n}(x)=H_{n}(x) / H_{n-1}(x)$. Then

$$
\mu_{n}(x)=\left(\frac{n+1}{n}\right)^{x-1} \frac{n}{n+x-1}=\left(1+\frac{1}{n}\right)^{x-1}\left(1+\frac{x-1}{n}\right)^{-1}
$$

By Lemma $1, \mu_{n}(x)>1$ for $x>2$ and $0<x<1$, while $\mu_{n}(x)<1$ for $1<x<2$.

At the points where the behaviour reverses, i.e. $x=1$ for $G_{n}(x)$ and $x=1$ and 2 for $H_{n}(x)$, we have already seen that the sequence in question is constant.

Convergence of Euler's limit is now an easy deduction:

Theorem 2: For all real $x$ except 0 and negative integers, $G_{n}(x)$ and $H_{n}(x)$ tend to a common limit as $n \rightarrow \infty$. Taking this limit as the definition of $\Gamma(x)$, we have $\Gamma(x+1)=x \Gamma(x)$.

Proof. First, fix $x$ with $1 \leqslant x \leqslant 2$. Then $G_{n}(x)$ is increasing, $H_{n}(x)$ is decreasing and $G_{n}(x) \leqslant H_{n}(x)$ for all $n$. Hence $G_{n}(x) \leqslant H_{1}(x)$ for all $n$, so $G_{n}(x)$ is bounded above, hence convergent. As already noted, it follows that $H_{n}(x)$ converges to the same limit.

To deduce the statement for all other $x$, just note that

$$
G_{n}(x+1)=\frac{n x}{n+x} G_{n}(x),
$$

so that if $G_{n}(x) \rightarrow L$, then $G_{n}(x+1) \rightarrow x L$ (and conversely). Repeated 
steps of length 1 (both forwards and backwards) now establish both convergence and $\Gamma(x+1)=x \Gamma(x)$.

The value $\Gamma(n)=(n-1)$ ! follows at once.

Euler's limit leads on to various identities that are basic in the theory of the gamma function, including Weierstrass's infinite product formula for $\Gamma(x)$ and the infinite series expressions for $\log \Gamma(x)$ and $\Gamma^{\prime}(x) / \Gamma(x)$. Also, at some point it is necessary to establish the equivalence of Euler's limit with the integral definition. All this can be seen in numerous books, e.g. those already mentioned. However, none of it is needed for our present purposes.

\section{Inequalities given by the proof of convergence}

If a sequence is increasing and convergent, then its terms are not greater than the limit. So for $1 \leqslant x \leqslant 2$, the reasoning in Theorem 2 also shows, with no further effort, that $G_{n}(x) \leqslant \Gamma(x) \leqslant H_{n}(x)$. Furthermore, these inequalities can be rewritten in two ways, both interesting. Since

$$
x(x+1) \ldots(x+n-1)=\frac{\Gamma(n+x)}{\Gamma(x)},
$$

we have

$$
\begin{gathered}
G_{n}(x)=n^{x-1} n ! \frac{\Gamma(x)}{\Gamma(n+x)}, \\
H_{n}(x)=(n+1)^{x-1} n ! \frac{\Gamma(x)}{\Gamma(n+x)} .
\end{gathered}
$$

Consider also the binomial coefficients

$$
K_{n}(x)=(-1)^{n}\left(\begin{array}{c}
-x \\
n
\end{array}\right)=\left(\begin{array}{c}
n+x-1 \\
n
\end{array}\right)=\frac{x(x+1) \ldots(x+n-1)}{n !} .
$$

This is the coefficient of $t^{n}$ in the series for $(1-t)^{-x}$. Clearly

$$
K_{n}(x)=\frac{n^{x-1}}{G_{n}(x)}=\frac{(n+1)^{x-1}}{H_{n}(x)} .
$$

The following system of inequalities now falls into our lap with no further work:

Theorem 3: For $1 \leqslant x \leqslant 2$ and integers $n \geqslant 1$,

$$
\begin{gathered}
G_{n}(x) \leqslant \Gamma(x) \leqslant H_{n}(x), \\
n^{x-1} n ! \leqslant \Gamma(n+x) \leqslant(n+1)^{x-1} n !, \\
\frac{n^{x-1}}{\Gamma(x)} \leqslant K_{n}(x) \leqslant \frac{(n+1)^{x-1}}{\Gamma(x)} .
\end{gathered}
$$

For $0 \leqslant x \leqslant 1$, all these inequalities are reversed. 
These inequalities are asymptotic, in the sense that the ratio of the quantities compared tends to 1 as $n$ tends to infinity. A further signal of their effectiveness is that in (2) and its reverse, the upper bounds are exact at both ends of the stated interval for $x$, and the lower bounds are exact at $x=1$. For example, when $x=1$, both bounds in (2) are $n$ !, equal to $\Gamma(n+1)$. The inequalities are strict except at these points.

Statement (2) amounts to a pair of inequalities for $\Gamma(y)$ for all $y>1$ : just express $y$ as $n+x$. Statement (3) can be viewed as a pair of bounds for $K_{n}(x)$, with $\Gamma(x)$ regarded as known.

Example 1: By (2), with $x=\frac{3}{2}$ and $n$ replaced by $n-1$, we have

$$
(n-1)^{1 / 2}(n-1) !<\Gamma\left(n+\frac{1}{2}\right)<n^{1 / 2}(n-1) ! \text {. }
$$

Example 2: We have

$$
G_{n}\left(\frac{1}{2}\right)=\frac{n^{-1 / 2} n !}{\frac{1}{2}\left(1+\frac{1}{2}\right) \ldots\left(n-\frac{1}{2}\right)}=\frac{2.4 \ldots(2 n)}{1.3 \ldots(2 n-1) n^{1 / 2}} .
$$

By the Wallis product, this converges to $\sqrt{\pi}$, so Euler's limit gives the value $\Gamma\left(\frac{1}{2}\right)=\sqrt{\pi}$. Furthermore, the statement $H_{n}\left(\frac{1}{2}\right) \leqslant \Gamma\left(\frac{1}{2}\right) \leqslant G_{n}\left(\frac{1}{2}\right)$ equates to the inequalities

$$
\frac{2.4 \ldots(2 n)}{1.3 \ldots(2 n-1)(n+1)^{1 / 2}} \leqslant \sqrt{\pi} \leqslant \frac{2.4 \ldots(2 n)}{1.3 \ldots(2 n-1) n^{1 / 2}} .
$$

Note on the case $x>2$ : For $x>2$, we clearly have in the same way $G_{n}(x) \leqslant H_{n}(x) \leqslant \Gamma(x)$, hence $(n+1)^{x-1} n !<\Gamma(n+x)$ and $(n+1)^{x-1} / \Gamma(x)<K_{n}(x)$. However, better lower bounds, as well as the missing upper bounds, are obtained by writing $x=r+y$, where $1<y<2$, and applying (2).

Note on $\Gamma_{n}(x)$ : Since $x \Gamma_{n}(x)=G_{n}(x+1)$, it follows from Theorem 1 that for all $x>0, \Gamma_{n}(x)$ increases with $n$ and $\Gamma_{n}(x) \leqslant \Gamma(x)$.

\section{Log-convexity and the Bohr-Mollerup theorem}

Recall that a function $f$ is 'convex' if it lies below the straight-line chords between pairs of points of its graph. Roughly speaking, this means that the graph is curving upwards. The precise definition is: if $x_{1}<x_{2}$ and $x_{\lambda}=(1-\lambda) x_{1}+\lambda x_{2}$, where $0 \leqslant \lambda \leqslant 1$, then

$$
f\left(x_{\lambda}\right) \leqslant(1-\lambda) f\left(x_{1}\right)+\lambda f\left(x_{2}\right) .
$$

We say that $f$ is 'concave' if $-f$ is convex.

In particular, if $f$ is convex and $f\left(x_{1}\right)=f\left(x_{2}\right)=b$ (say), then $f(x) \leqslant b$ for $x_{1}<x<x_{2}$. Further, $f(x) \geqslant b$ for $x<x_{1}$ and for $x>x_{2}$ : if we had $f(x)<b$ for some $x>x_{2}$, then we would have $f\left(x_{2}\right)<b$, since $x_{2}$ is between $x_{1}$ and $x$. 
It is a well-known consequence of the mean-value theorem that if $f^{\prime}(x)$ is increasing on some interval $I$, then $f(x)$ is convex there.

If $f(x)$ is positive, then the function $\log f(x)$ is convex if, in the above notation,

$$
f\left(x_{\lambda}\right) \leqslant f\left(x_{1}\right)^{1-\lambda} f\left(x_{2}\right)^{\lambda} .
$$

Using the fact that the exponential function is convex and increasing, it is easy to show that convexity of $\log f(x)$ implies convexity of $f(x)$ (we leave the details as an exercise for the reader), but the converse is not true: $x^{2}$ is convex, while $\log x^{2}=2 \log x$ is concave.

For the gamma function, this property follows very easily from Euler's limit expression:

Theorem 4: The function $\log \Gamma(x)$ is convex for $x>0$.

Proof: For $x>0$, we have

$$
\log G_{n}(x)=x \log n+\log |(n-1) !|-\sum_{r=0}^{n-1} \log (x+r),
$$

hence

$$
\frac{d}{d x} \log G_{n}(x)=\log n-\sum_{r=0}^{n-1} \frac{1}{x+r} .
$$

This is an increasing function of $x$, since $1 /(x+r)$ is decreasing. Hence $\log G_{n}(x)$ is convex. Now if $f_{n}(x)$ is convex for each $n$ and $f_{n}(x) \rightarrow f(x)$ as $n \rightarrow \infty$ (for each $x$ ), then it follows at once from the definition that $f(x)$ is convex. Hence $\log \Gamma(x)$ is convex.

The right-hand inequality in Example 1 reflects this property, since it equates to $\Gamma\left(n+\frac{1}{2}\right) \leqslant \Gamma(n)^{1 / 2} \Gamma(n+1)^{1 / 2}$.

We mention in passing that to prove Theorem 4 from the integral definition of the gamma function, one needs the integral version of Hölder's inequality.

The Bohr-Mollerup theorem states that, conversely, the gamma function is the only function (apart from constant multiples) that has this property together with $f(x+1)=x f(x)$. It was first announced, not in a research article, but in the textbook [6], which appeared in 1922 and had a deep influence on the development of mathematics in Denmark. Harald Bohr was the brother of the famous physicist Niels Bohr, and played in a rather successful Danish national football team before going on to become a distinguished mathematician. The exact statement of the theorem is as follows.

Theorem 5: Suppose that $f(x)$ is defined for $x>0$ and: (i) $\log f(x)$ is convex; (ii) $f(x+1)=x f(x)$; (iii) $f(1)=1$. Then $f(x)=\Gamma(x)$. 
We present a version of the simple and elegant proof given in Artin's classic short book [7]. By a neat reversal of our previous reasoning, we show that these conditions imply that $f(x)$ satisfies inequality (2), which leads straight back to Euler's limit. The main step is the following result:

Theorem 6: Let $f$ be as in Theorem 5. For positive $a$ and $x$, write $R(a, x)=f(a+x) / f(a)$. Then

$$
\begin{array}{ll}
R(a, x) \leqslant a^{x} & \text { for } 0 \leqslant x \leqslant 1, \\
R(a, x) \geqslant a^{x} & \text { for } x \geqslant 1 .
\end{array}
$$

Proof: Let $F(x)=\log f(a+x)-x \log a$. We show that $F(x)$ is convex. Let $x_{\lambda}=(1-\lambda) x_{1}+\lambda x_{2}$. Then $a+x_{\lambda}=(1-\lambda)\left(a+x_{1}\right)+\lambda\left(a+x_{2}\right)$, so

$$
\begin{aligned}
F\left(x_{\lambda}\right) & =\log f\left(a+x_{\lambda}\right)-x_{\lambda} \log a \\
& \leqslant(1-\lambda) \log f\left(a+x_{1}\right)+\lambda \log f\left(a+x_{2}\right)-\left[(1-\lambda) x_{1}+\lambda x_{2}\right] \log a \\
& =(1-\lambda) F\left(x_{1}\right)+\lambda F\left(x_{2}\right),
\end{aligned}
$$

as required. Now by property (ii),

$$
F(1)=\log f(a+1)-\log a=\log f(a)=F(0) .
$$

By the remark following the definition of convexity, it follows that $F(x) \leqslant \log f(a)$ for $0 \leqslant x \leqslant 1$ and $F(x) \geqslant \log f(a)$ for $x \geqslant 1$. This equates to (4) and (5).

Corollary: For $f$ as in Theorem 5, we have

$$
R(a, x) \leqslant a(a+1)^{x-1} \quad \text { for } 1 \leqslant x \leqslant 2 .
$$

Proof: For $1 \leqslant x \leqslant 2$, we have

$$
R(a+1, x-1)=\frac{f(a+x)}{f(a+1)} \leqslant(a+1)^{x-1},
$$

so that $f(a+x) \leqslant a(a+1)^{x-1} f(a)$.

Proof of Theorem 5: Conditions (ii) and (iii) clearly imply that $f(n)=(n-1)$ ! for positive integers $n$. Because of (ii), it is enough to prove the statement for $1<x<2$. By (5) and (6), we then have, for integers $n$,

$$
n^{x} \leqslant \frac{f(n+x)}{f(n)} \leqslant n(n+1)^{x-1},
$$

so

$$
n^{x-1} n ! \leqslant f(n+x) \leqslant(n+1)^{x-1} n ! .
$$


In other words, $f(x)$ satisfies (2). Since $f(n+x)=x(x+1) \ldots(x+n-1) f(x)$, this equates to

$$
G_{n}(x) \leqslant f(x) \leqslant H_{n}(x)
$$

Since $G_{n}(x)$ and $H_{n}(x)$ both converge to $\Gamma(x)$, it follows that $f(x)=\Gamma(x)$.

Of course, we have actually shown that (2) applies to $f(x)$ (hence to $\Gamma(x)$ ) with the integer $n$ replaced by any $a>0$. By further substitutions of the type used in the corollary, one can go on to derive a systematic scheme of inequalities for the ratio $\Gamma(a+x) / \Gamma(a)$, applying in tum for $0 \leqslant x \leqslant 1$, for $1 \leqslant x \leqslant 2$ and for $x \geqslant 2$. Interestingly, some of these inequalities were rediscovered by later writers unaware of Artin's proof, using more elaborate methods. A survey of these results, and some of the history, is given in [8].

\section{References}

1. David Fowler, A simple approach to the factorial function, Math. Gaz. 80 (November 1996) pp. 378-381.

2. David Fowler, A simple approach to the factorial function - the next step, Math. Gaz. 83 (March 1999) pp. 53-57.

3. E. T. Whittaker and G. N. Watson, A course of modern analysis (4th edn.), Cambridge University Press (1965).

4. E. T. Copson, An Introduction to the theory of functions of a complex variable, Oxford University Press (1935).

5. George E. Andrews, Richard Askey and Ranjam Roy, Special Functions, Cambridge University Press (1999).

6. H. Bohr and J. Mollerup, Laerebog i Matematisk Analyse, Gjellerup, Copenhagen (1922).

7. Emil Artin, Einfuihrung in die Theorie der Gammafunktion, Teubner, Leipzig (1931); English translation: The gamma function, Holt, Rinehart and Winston (1964).

8. G. J. O. Jameson, Inequalities for gamma function ratios, Amer. Math. Monthly 120 (2013) pp. 936-940.

G. J. O. JAMESON

Dept. of Mathematics and Statistics, Lancaster University, Lancaster LA1 4YF e-mail: g.jameson@lancaster.ac.uk 